\title{
AVALIAÇÃO DE SISTEMAS MICELARES DE DUAS FASES AQUOSAS COMPOSTOS POR TRITON X-114 E LÍQUIDOS IÔNICOS COMO ADJUVANTES NA EXTRAÇÃO DE COLORANTES
}

\author{
B.R. ZACCARIM ${ }^{1}$, P. A.M. NASCIMENTO ${ }^{1}$, M.F.S. TEIXEIRA ${ }^{2}$, J.F.B PEREIRA ${ }^{1}$ e V.C. \\ SANTOS-EBINUMA ${ }^{1}$ \\ ${ }^{1}$ UNESP - Universidade Estadual Paulista "Júlio de Mesquita Filho", Departamento \\ de Bioprocessos e Biotecnologia, Escola de Ciências Farmacêuticas \\ ${ }^{2}$ UFAM - Universidade Federal do Amazonas, Departamento de Parasitologia \\ E-mail para contato: bruzaccarim@hotmail.com
}

\begin{abstract}
RESUMO - Os líquidos iônicos (LIs) têm ganhado grande importância nas áreas da biotecnologia como solventes alternativos aos orgânicos, uma vez que a demanda por tecnologias mais limpas tem aumentado. Dentro deste tema, LIs podem ser adicionados a sistemas micelares de duas fases aquosas (SMDFA) para melhorar a extração de biomoléculas por esta técnica. SMDFA apresentam habilidade de manter as conformações e atividades das biomoléculas de interesse enquanto migram de fase sendo de grande interesse para extrair biomoléculas do meio fermentado. Assim, o objetivo deste trabalho foi avaliar e comparar a extração de colorantes naturais vermelhos produzidos por cultivo submerso de $T$. amestolkiae utilizando SMDFA composto pelo surfactante não-iônico TX-114 com cloreto de colina([Ch]Cl) e butanoato de colina ([Ch]But) como adjuvantes. Foram realizados experimentos para determinação das curvas binodais dos LIs e, posteriormente, experimentos de partição com os sistemas já caracterizados. $\mathrm{O}$ melhor sistema foi aquele que continha butanoato de colina em sua composição.
\end{abstract}

\section{INTRODUÇÃO}

O mercado de colorantes é constituído pelos colorantes naturais e artificiais, sendo que atualmente há uma ampla tendência da substituição dos artificiais pelos naturais. Estes são obtidos de plantas, insetos (Boo et al., 2012; Deveoglu et al., 2012; Unagul et al., 2005) e microrganismos (Cho et al., 2002; Jiang et al., 2005; Méndez et al., 2011; Yang et al., 2009), sendo que os últimos são os principais utilizados para a sua produção. Dentro deles, os fungos filamentosos possuem vasta versatilidade metabólica, o que possibilita o emprego dos mesmos em diversos processos industriais, como a produção de ácidos orgânicos, polissacarídeos, enzimas, alcaloides, antibióticos e colorantes (Babitha et al., 2007; ElEnshasy, 2007; Velmurugan et al., 2010).

Em pesquisas realizadas por Teixeira et al. (2012), T. amestolkiae mostrou ser potencial produtor de colorantes naturais com alta atividade antimicrobiana e ausência de toxicidade contra o microcrustáceo Artemia salina. Em outros estudos, os colorantes 
vermelhos produzidos por este microrganismo mostraram estabilidade em condições alcalinas, na presença de sais e polímeros (Santos-Ebinuma et al., 2013a), além de apresentarem atividade antioxidante.

Além da etapa de produção, desenvolver técnicas de extração/purificação mais econômicas, de forma a incrementar os parâmetros de purificação e ao mesmo tempo reduzir os custos operacionais (Pessoa Jr. e Kilikian, 2005) são de interesse. Neste contexto, pode-se citar os SMDFA, os quais são formados por agentes tensoativos, que são moléculas anfifílicas (Cordisco et al., 2015). Os tensoativos em concentrações acima da concentração micelar crítica (CMC) formam agregados, conhecidos como micelas (Santos-Ebinuma et al., 2013a), e o aumento da temperatura provoca o fenômeno de separação de fases, no qual uma fase é rica em micelas e a outra pobre em micelas (Hagas et al., 2013) Todo o processo é dependente da concentração e estrutura do agente tensoativo, da presença de aditivos, da força iônica e pH (Liu et al, 1998).

A inserção de LIs nos SMDFA pode incrementar o processo de extração de biomoléculas, pois podem contribuir para a alteração das propriedades físico-químicas de micelas surfactantes (Vicente et al., 2014). Os LIs são sais orgânicos que se encontram em estado líquido, em condições atmosféricas normais de temperatura e pressão, e apresentam características únicas como, estabilidade térmica e química, baixo ponto de fusão, elevada força de solvatação e ampla gama de estruturas químicas (Freire et al., 2015). Diante do exposto, este trabalho visou a extração de colorantes naturais produzidos por Talaromyces amestolkiae empregando SMDFA com LIs como adjuvantes.

\section{MATERIAL E MÉTODOS}

O LI butanoato de colina foi sintetizado pelo grupo de pesquisa. O tensoativo nãoiônico Triton X-114 e o LI cloreto de colina foram adquiridos da Sigma-Aldrich ${ }^{\mathrm{TM}}$. Todos os outros reagentes foram de grau analítico.

\subsection{Microrganismo e produção dos colorantes naturais}

T. amestolkiae (antigamente $P$. purpurogenum) foi cedido pela Coleção de Culturas DPUA da Universidade Federal do Amazonas - UFAM. A cultura foi preservada em água destilada esterilizada e foi reativada em caldo glicosado de acordo com Lacaz et al. (2002) em agitador rotativo a $30{ }^{\circ} \mathrm{C}$, durante 7 dias e depois transferidos para tubos inclinados contendo meio CYA (ágar extrato de levedura Czapec), os quais foram incubados a $30^{\circ} \mathrm{C}$, por 7 dias, e, posteriormente, conservados em geladeira a $4^{\circ} \mathrm{C}$, sendo repicados a cada 30 dias.

Para os experimentos de produção, o inóculo foi realizado em placa de Petri contendo meio BDA (ágar batata dextrose) suplementado com $0,5 \%(\mathrm{~m} / \mathrm{v})$ de extrato de levedura por um período de 7 dias a $30^{\circ} \mathrm{C}$, o qual foi inoculado ( 10 discos de micélio de $8 \mathrm{~mm}$ de diâmetro) em frascos Erlenmeyers $(250 \mathrm{~mL})$ contendo $50 \mathrm{~mL}$ de meio de cultivo. O cultivo submerso foi conduzido em agitador orbital por 168 horas a $30^{\circ} \mathrm{C}$. A composição do meio de cultivo foi de $10 \mathrm{~g} / \mathrm{L}$ de peptona de carne, $30 \mathrm{~g} / \mathrm{L}$ de glicose e $1 \mathrm{~g} / \mathrm{L}$ de extrato de carne, com pH inicial de 7. Ao fim da produção, o meio contendo os colorantes naturais foi filtrado em papel de filtro Whatman $\mathrm{n}^{\circ} 1$. 


\subsection{Construção e caracterização das curvas binodais}

Foram construídas as curvas binodais para os sistemas através do método do "ponto de névoa" de acordo com Blankschtein et al. (1986). Os estudos foram realizados em sistemas formados por Triton X-114 em diferentes concentrações $(1-15,0 \% \mathrm{~m} / \mathrm{m})$ em $\mathrm{H}_{2} \mathrm{O}$ e na presença dos LIs nas concentrações de $0,05,0,1$ e $0,5 \mathrm{M}$.

\subsection{Sistemas de extração}

Os sistemas de extração foram preparados nas mesmas concentrações empregadas anteriormente em tubos de ensaio graduados de $15 \mathrm{~mL}$, pela adição por gravimetria de Triton $\mathrm{X}-114$, meio fermentado, $\mathrm{LI}$ e $\mathrm{H}_{2} \mathrm{O}$ nas concentrações desejadas. $\mathrm{O}$ sistema foi homogeneizado em agitador orbital a $8 \mathrm{rpm}$ por 30 minutos. Em seguida, transferido para banho termorregulado com a temperatura previamente ajustada ao desejado e mantido em repouso por 3 horas. Após o repouso, os sistemas foram centrifugados e amostras das fases superior (top) e inferior (bottom) foram coletadas cuidadosamente, com o auxílio de seringa e agulha. Cada experimento de partição foi feito em triplicata e os respectivos desvios padrões e intervalos de confiança calculados. Em ambas as fases foram determinadas a concentração de colorantes naturais a fim de se calcular os parâmetros de extração: coeficiente de partição do colorante $(\mathrm{K})$ - Equação 1, recuperação do colorante nas fase inferior $\left(\mathrm{R}_{\mathrm{F}}\right)$ - Equação 2 e razão volumétrica $\left(\mathrm{R}_{\mathrm{V}}\right)$ - Equação 3 .

$$
\begin{aligned}
& K_{P}=\frac{C_{T}}{C_{F}} \\
& R_{F}=\frac{100}{1+R_{V} K} \\
& R_{V}=\frac{V_{F}}{V_{T}}
\end{aligned}
$$

Em que: $C_{T}$ e $C_{F}$ referem-se às concentrações do colorante (UA) na fase de topo (rica em micelas) e fundo (pobre em micelas), respetivamente. $R_{V}$ representa a razão volumétrica entre o volume da fase de topo $\left(V_{T}\right)$ e o volume da fase de fundo $\left(V_{F}\right)$.

\section{RESULTADOS E DISCUSSÃO}

A Figura 1 apresenta as curvas binodais construídas a partir das temperaturas obtidas no experimento do ponto de névoa. 
Figura 1 - Curvas binodais dos SMDFA formados por Triton X-114 (TX-114) na presença ou ausência de LI $(\multimap 0,05 \mathrm{M} ; \multimap-0,1 \mathrm{M} ; \multimap-0,5 \mathrm{M}$ para os sistemas com cloreto de colina, $\rightarrow-0,05 \mathrm{M} ;-0,1 \mathrm{M} ; \rightarrow 0,5 \mathrm{M}$ para os sistemas com butanoato de colina e ausência de LI)

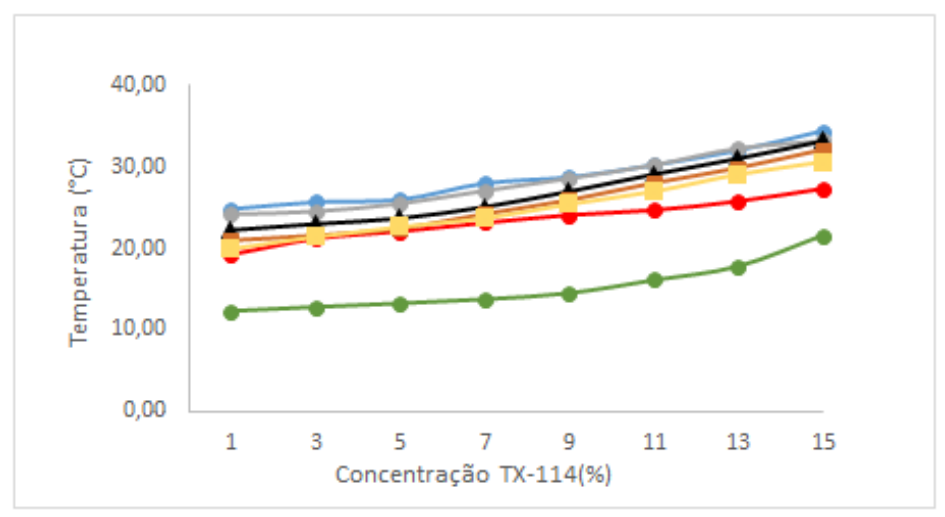

A partir destes resultados foi possível observar que as menores temperaturas foram obtidas com o sistema contendo butanoato de colina na concentração de $0,5 \mathrm{M}$ e as maiores foram encontradas nas amostras contendo cloreto de colina nas concentrações de $0,05 \mathrm{M}$ e $0,1 \mathrm{M}$, sendo que as últimas foram as únicas curvas acima da encontrada no experimento realizado sem LI. Uma possível explicação para este fenômeno é que o aumento da cadeia carbônica dos compostos os deixam mais hidrofóbicos, requerendo, dessa forma, menor hidratação e, consequentemente, menor quantidade de energia necessária para a separação do sistema em duas fases (o que resulta em menores temperaturas de separação).

Além disso, de acordo com a atuação dos ións da Série de Hofmeister, o cloreto pode ser classificado como cosmotrópico, ou seja, aumenta a estruturação das moléculas de água ao redor do íon (Machado, 2010) por possuir maior interação com estas (Lo Nostro et al., 2006). Assim, a maior hidratação acaba requerendo maior energia para a separação de fases do sistema e então maiores temperaturas são encontradas neste sistema.

A Figura 2 apresenta os coeficientes de partição do colorante vermelho para os ensaios contendo LI. 
Figura 2 - Coeficientes de partição obtidos para a partição de colorantes naturais vermelhos por SMDFA composto por Triton X-114 (TX-114) na presença de LI. ( $\square$ 0,05M; $0,1 \mathrm{M} ;=0,5 \mathrm{M}$ para os sistemas com cloreto de colina, $\square 0,05 \mathrm{M} ;=0,1 \mathrm{M} ;=0,5 \mathrm{M}$ para os sistemas com butanoato de colina)

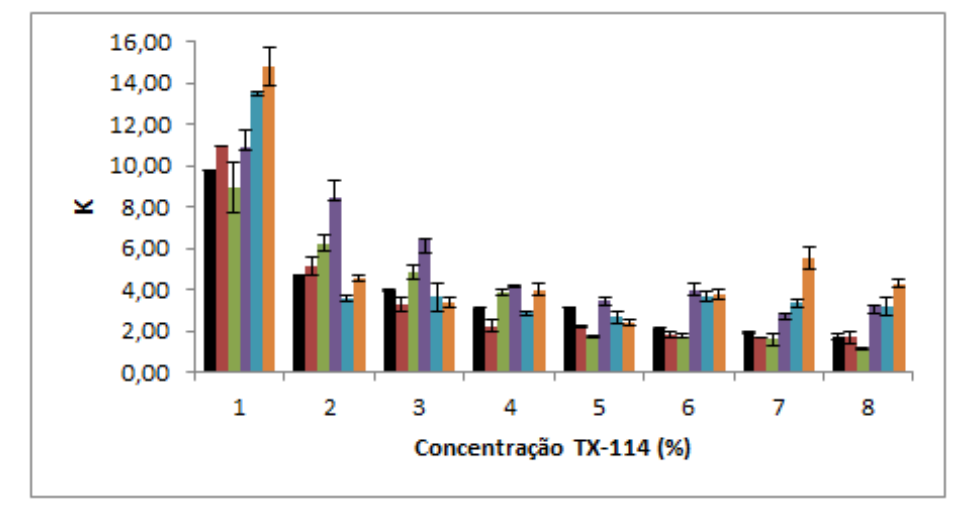

Pelo gráfico, foi possível concluir que a partição do colorante foi eficiente e que migrou mais para a fase bottom (fase rica em surfactante), uma vez que todos os valores encontrados ficaram acima de 1 . Notou-se também que para menores concentrações de Triton X-114 a partição foi mais efetiva. Dentro dos melhores resultados, os ensaios com cloreto de colina mostraram-se mais vantajosos. Ademais, além da separação do colorante, houve também a concentração do mesmo nos ensaios em que o valor do coeficiente de partição obtido foi bem maior que 1 .

As Tabelas 1 e 2 apresentam as recuperações do colorante na fase bottom com os sistemas utilizado cloreto de colina e butanoato de colina, respectivamente.

Tabela 1 - Eficiência de extração na fase bottom para os sistemas com cloreto de colina.

\begin{tabular}{|c|c|c|c|}
\hline TX-114 & {$[\mathrm{Ch}] \mathrm{Cl} 0,05 \mathrm{M}$} & {$[\mathrm{Ch}] \mathrm{Cl} 0,1 \mathrm{M}$} & {$[\mathrm{Ch}] \mathrm{Cl} 0,5 \mathrm{M}$} \\
\hline \hline $1 \%$ & 42,29 & 59,99 & 62,15 \\
\hline $3 \%$ & 85,65 & 57,57 & 84,70 \\
\hline $5 \%$ & 82,05 & 75,77 & 75,85 \\
\hline $7 \%$ & 82,61 & 74,06 & 80,14 \\
\hline $9 \%$ & 84,91 & 85,23 & 73,22 \\
\hline $11 \%$ & 79,85 & 78,64 & 76,60 \\
\hline $13 \%$ & 82,61 & 82,42 & 76,41 \\
\hline $15 \%$ & 88,72 & 86,57 & 77,12 \\
\hline
\end{tabular}

Tabela 2 - Eficiência de extração na fase bottom para os sistemas com butanoato de colina.

\begin{tabular}{|c|c|c|c|}
\hline TX-114 & [Ch]But 0,05M & [Ch]But 0,1M & [Ch]But 0,5M \\
\hline \hline $1 \%$ & 85,27 & 85,92 & 62,38 \\
\hline $3 \%$ & 98,57 & 97,29 & 95,74 \\
\hline $5 \%$ & 98,85 & 99,43 & 96,96 \\
\hline $7 \%$ & 99,91 & 99,75 & 98,25 \\
\hline $9 \%$ & 99,20 & 99,90 & 98,80 \\
\hline $11 \%$ & 98,87 & 99,56 & 99,36 \\
\hline $13 \%$ & 99,71 & 99,71 & 99,64 \\
\hline $15 \%$ & 99,63 & 99,68 & 99,82 \\
\hline
\end{tabular}


A partir da análise dos dados das tabelas, foi possível notar que as maiores eficiências de extração na fase bottom foram obtidas nos ensaios que continham butanoato de colina e que os melhores resultados foram similares nas concentrações de 7-15\%. Dessa forma, notouse grade importância da maior cadeia carbônica deste LI quando comparado ao outro utilizado.

\section{CONCLUSÃO}

A extração de colorantes naturais utilizando líquidos iônicos como adjuvantes nos sistemas micelares de duas fases aquosas mostrou-se eficiente, uma vez que as eficiências de extração encontradas foram superiores a $50 \%$ em todos os ensaios. As amostras contendo butanoato de colina mostraram-se mais efetivas, obtendo valores próximos a $100 \%$, sem apresentar diferenças no aumento da concentração do líquido iônico. Este LI também é de interesse, pois, mesmo apresentando coeficientes de partição ligeiramente menores que o cloreto de colina, apresentou menores temperaturas de partição, o que gera uma possível economia para o processo. Dessa forma, concluiu-se que o LI butanoato de colina tem grande potencial para a extração de colorantes naturais.

\section{AGRADECIMENTOS}

Os autores agradecem à Fundação de Amparo à Pesquisa do Estado de São Paulo (FAPESP-Processo 2014/01580-3), CNPq e CAPES.

\section{REFERÊNCIAS}

Babitha, S.; Soccol, C.R.; Pandey, A. Solid-state fermentation for the production of Monascus pigments from jackfruit seed. Bioresource Technology, v.98, n.8, p.1554-1560, 2007.

Blankschtein, D.; Thurston, G.M.; Benedek, G.B. Journal Chemical Physics, v.85, p.72687288, 1986

Boo, H.-O.; Hwang, S.-J.; Bae, C.-S.; Park, S.-H.; Heo, B.-G.; Gorinstein, S. Extraction and characterization of some natural plant pigments. Industrial Crops and Products, v.40, p.129135, 2012.

Cho, Y.J.; Park, J.P.; Hwang, H.J.; Kim, S.W.; Choi, J.W.; Yun, J.W. Production of red pigment by submerged culture of Paecilomyces sinclairii. Letters in Applied Microbiology, v.35, n.3, p.195-202, 2002.

Cordisco, E.; Haidar, C.N.; Goñi, R.; Nerli, B.B.; Malpiedi, L.P. Fluid Phase Equilibria, v.393, p. 111- 116, 2015.

Deveoglu, O.; Cakmakci, E.; Taskopru, T.; Torgan, E.; Karadag, R. Identification by RPHPLC-DAD, FTIR, TGA and FESEM-EDAX of natural pigments prepared from Datisca cannabina L. Dyes and Pigments, v.94, n.3, p.437-442, 2012. 
El-Enshasy, H.A. Filamentous fungal cultures - process characteristics, products, and applications. In: YANG, S.-T., eds. Bioprocessing for value-added products from renewable resources: new technologies and applications. Amsterdam: Elsevier, 2007. cap.9, p.225-261.

Freire, M.G.; Cláudio, A.F.M.; Araújo, J.M.M.; Coutinho, J.A.P.; Marrucho, I.M.; Lopes, J.N.C; Rebelo, L.P.N. Chem. Soc. Rev., v.41, p. 4966- 4995, 2012.

Haga, R.B.; Santos-Ebinuma, V.C; Silva, M.S.C.; Pessoa Jr, A.; Rangel-Yagui, C.O. Separation and Purification Technology, v.103, p. 273-278, 2013.

Jiang, Y.; Li, H.B.; Chen, F.; Hyde, K.D. Production potential of water-soluble Monascus red pigment by a newly isolated Penicillium sp. Journal of Agricultural Technology, v.1, p.113$126,2005$.

Lacaz, C.S.; Porto, E.; Marins, J.E.C.; Heins-Vacarri, E.M.; Mello, N.K. Tratado de micologia médica Lacaz. 9.ed. São Paulo: Sarvier, 2002. 1104p

Liu, C-L.; Kamei, D.T.; King, J.A.; Wang, D.I.C.; Blankschtein, D. Journal of Chromatography B, v.711, p.127-138, 1998.

Lo Nostro, P.; Ninham, B.W.; Milani, S.; Lo Nostro, A.; Pesavento, G.; Baglioni, P. Hofmeister effects in supramolecular and biologival systems. Biophysical Chemistry. V 124, p.208-213, 2006.

Machado D.C.; Estudo da influência de substâncias cosmotrópicas e caotrópicas na interação de moléculas unitárias orgânicas com nanoporos individuais protéicos. Tese (Mestrado em Ciências Biológicas). Universidade Federal de Pernambuco. Recife, 2010.

Méndez, A.; Pérez, C.; Montañéz, J.C.; Martínez, G.; Aguilar, C.N. Red pigment production by Penicillium purpurogenum $\mathrm{GH} 2$ is influenced by $\mathrm{pH}$ and temperature. Journal of Zhejiang University, Science, B, v.12, n.12, p.961-968, 2011.

Pessoa Jr., A., Kilikian, B.V. Purificação de produtos biotecnológicos. Barueri: Manole, 2005. 444p.

Santos-Ebinuma, V.C.; Lopes, A.M.; Converti, A.; Pessoa Jr, A.; Yagui, C.O.R. Fluid Phase Equilibria, v.360, p. 435- 438, 2013.

Teixeira, M.F.S.; Martins, M.S.; Silva, J.C.; Kirsch, L.S.; Fernandes, O.C.C.; Carneiro, A.L.B.; Conti, R.; Durán, N. Amazonian biodiversity: pigments from Aspergillus and Penicillium-characterizations, antibacterial activities and their toxicities. Current Trends in Biotechnology and Pharmacy, v.6, n.3, p.300-311, 2012.

Unagul, P.; Wongsa, P.; Kittakoop, P.; Intamas, S.; Srikitikulchai, P.; Tanticharoen, M. Production of red pigments by the insect pathogenic fungus Cordyceps unilateralis BCC 1869. Journal of Industrial Microbiology \& Biotechnology, v.32, n.4, p.135-140., 2005. 
Velmurugan, P; Hur, H; Balachandar, V.; Kamala-Kanna, S.; Lee, K.; Lee, S.; Chae, J.; Shea, P.J.; OH, B. Monascus pigment production by solid-state fermentation with corn cob substrate. Journal of bioscience and bioengineering, v. 112, n. 6, p. 590-594, 2011.

Vicente, F.A.; Malpiedi, L.P.; Silva, F.A.; Pessoa Jr., A.; Coutinho, J.A.P.; Ventura, S.P.M. Separation and Purification Technology, v.135, p. 259- 267, 2014.

Yang, H.; Xiao, C.; Ma, W.; He, G. The production of hypocrellin colorants by submerged cultivation of the medicinal fungus Shiraia bambusicola. Dyes and Pigments, v.82, n.2, p.142146, 2009. 